Jahrbuch Schweiz - Dritte Welt 1989

\title{
II. Innenpolitik
}

\section{(2) OpenEdition \\ 1 Journals}

Electronic version

URL: http://journals.openedition.org/sjep/1119

DOI: $10.4000 /$ sjep. 1119

ISSN: 1663-9677

\section{Publisher}

Institut de hautes études internationales et du développement

\section{Printed version}

Date of publication: 1 décembre 1989

Number of pages: 65-71

ISSN: 1660-5926

\section{Electronic reference}

«II. Innenpolitik», Schweizerisches Jahrbuch für Entwicklungspolitik [Online], 8 | 1989, Online erschienen am: 18 März 2013, abgerufen am 08 September 2020. URL : http://journals.openedition.org/sjep/1119 ; DOI : https://doi.org/10.4000/sjep.1119

(c) The Graduate Institute 


\section{Innenpolitik}

\section{Nord/Süd-Kampagne}

Im ersten Halbjahr 1988 hat sich in allen 21 dem Europarat angeschlossenen Ländern eine Nord/Süd-Kampagne abgewickelt. Beschlossen wurde diese an einer Konferenz des Europarates in Lissabon 1984. Die Kampagne stand unter dem Zeichen der gegenseitigen Abhängigkeit und Solidarität. In der Schweiz wurden die Aktivitäten von einer nationalen Kommission organisiert und koordiniert. Eine Interpellation der Kommission für auswärtige Angelegenheiten gab Anlass zu einer breiten entwicklungspolitischen Debatte im Parlament.

Ziel der Kampagne war es, jene Kreise der Bevölkerung für die Probleme der Beziehungen zwischen den Entwicklungs- und den Industrieländern zu sensibilisieren, die auf dem üblichen Informationsweg und auch von den Entwicklungshilfeorganisationen noch nicht erreicht worden sind, insbesondere auch die Jugendlichen. Auftakt der Kampagne war eine Debatte über den Nord/Süd-Dialog im Januar 1988 im Europarat in Strassburg. Es folgten Kolloquien zu einzelnen Themen wie Schulden, Arbeitslosigkeit, Umwelt, Entwicklungshilfe und Kultur.

Abschluss der europäischen Kampagne bildete eine parlamentarische Konferenz - mit der Teilnahme von zahlreichen nichtstaatlichen Organisationen vom 1. bis 3. Juni 1988 in Madrid. Die Schweiz war ebenfalls vertreten; Konferenzteilnehmer war u.a. Ständerat Miville (SP/BS), welcher dem Rat in der Junisession Bericht erstattete.

Im Zentrum der Debatten in Madrid standen insbesondere folgende Themenkreise:

- Einschränkung der Landwirtschaftsproduktion der Industrieländer sowie der Subventionierung und des Protektionismus im Agrarsektor. 
- Einen offenen gerechten Handel mit den Entwicklungsländern fördern und ihnen den Zugang zu europäischen Märkten ermöglichen.

- Die Entwicklungsländer in ihren Bemühungen für die Umwelterhaltung unterstützen.

- Kampf gegen Rassismus, gegen Apartheid, für Menschenrechte und für Demokratie.

- Schuldenerleichterungen.

Die Konferenz hielt fest, dass die Zusammenarbeit zwischen den Nationen des Nordens und des Südens eine "politische Notwendigkeit» sei. Die Stagnation der wirtschaftlichen Entwicklung nach 1973, die hohe Arbeitslosigkeit, das starke Bevölkerungswachstum, der Wirtschaftsprotektionismus industrialisierter Länder, der Preissturz der Rohstoffe sowie die Umweltzerstörung haben die Entwicklungsperspektiven der Dritten Welt ernsthaft in Frage gestellt und die vorher erzielten Fortschritte im Kampf gegen die Armut weitgehend rückgängig gemacht, hält die «Erklärung von Madrid», das Schlussdokument der Konferenz, fest. Die Konferenzteilnehmer betonten die Wichtigkeit der Selbsthilfe und Selbstversorgung der Entwicklungsländer. Zur Verbesserung der Lage schlugen sie konkrete Massnahmen vor. Dazu gehört die Erfüllung des UNO-Auftrages, 0,7 Prozent des Bruttosozialprodukts für Entwicklungshilfe einzusetzen.

Ständerat Flückiger (FDP/JU), welcher ebenfalls als Mitglied der Schweizer Delegation in Madrid weilte, vermeinte in den alt bekannten Erklärungen und Entschliessungen das schlechte Gewissen Europas über das Nord/Süd-Gefälle zu verspüren, welches seine Ursachen nicht zuletzt im Kolonialismus und Neokolonialismus hat. Er nahm in der Berichterstattung im Ständerat die Frage der Entschuldungsmassnahmen auf und erwähnte den Rückkauf der bis auf zehn Prozent abgeschriebenen bolivianischen Schulden - die Schweiz hat sich an der Aktion beteiligt - als möglichen Ausweg aus der Schuldenkrise.

\section{Frauensymposium in Barcelona}

Am 30. und 31. Mai 1988 fand in Barcelona im Rahmen der Europaratskampagne ein Symposium zum Thema "Frauen und Entwicklung" statt. Am Symposium nahmen die Teilnehmerinnen aus Nord und Süd die Forderungen der UNFrauenkonferenz von Nairobi 1985 auf und debattierten über die spezifische Rolle und Stellung der Frauen in den Nord/Süd-Beziehungen. Die Themenbereiche waren: gegenseitige wirtschaftliche Abhängigkeit, Entwicklungszusammenarbeit, ökologische und sozio-kulturelle Interdependenz und Frieden. Die Tagung schloss mit Empfehlungen an die Konferenz von Madrid. Unter anderem wurde vorgeschlagen, die Auswirkungen der Kampagne regelmässig zu erfassen und im Europarat zu diskutieren. 


\section{Nationale Kampagne/Parlamentsdebatte}

Eine Interpellation der Kommission für auswärtige Angelegenheiten vom 29. März 1988 forderte den Bundesrat zu einer Erklärung vor dem Parlament über seine Politik im Rahmen der Nord/Süd-Beziehungen auf. Die Kommission hatte eine Erklärung vor der Vereinigten Bundesversammlung in der Märzsession verlangt, was aber abgewiesen wurde. In der Folge fand - angeregt durch die Interpellation - die Nord/Süd-Debatte in den einzelnen Räten statt, und zwar in der Junisession bei der Beratung des Geschäftsberichts des Bundesrates.

Bundesrat Felber erklärte, dass im Zusammenhang mit den Entwicklungsländern immer von Hilfe und Unterstützung, jedoch zuwenig von Dialog und Austausch die Rede sei. Der Bundesrat begrüsse nun die Möglichkeit, durch die Kampagne des Europarates die Beziehungen zwischen Industrie- und Entwicklungsländern im umfassenden Sinne, d.h. im politischen, wirtschaftlichen, ökologischen und kulturellen Zusammenhang zu diskutieren. Die Schweiz sei sich ihrer Verantwortung angesichts der prekären wirtschaftlichen Lage, in der sich zahlreiche Entwicklungsländer befinden, bewusst. Der Bundesrat strebe eine qualitative und quantitative Verbesserung der öffentlichen Entwicklungshilfe an. Zahlreiche Rednerinnen und Redner ergriffen das Wort. Die SP-Sprecherin (Pitteloud, SP/ND) vertrat die Auffassung, dass die schweizerische Entwicklungshilfe in erster Linie der eigenen Wirtschaft diene. In Fragen der Kapitalflucht etwa könne die Schweiz den betroffenen Entwicklungsländern entgegenkommen und die notwendigen Massnahmen zur Rückführung der Gelder treffen. Griffigere Gesetze für die Banken sowie für die Waffenausfuhr verlangte die Sprecherin der Grünen (Bär, G/BE). Allgemein war man sich im Parlament über die Notwendigkeit einig, den Kampf gegen die Armut und die Unterentwicklung zu verstärken. Dabei sei die globale Zusammenarbeit unerlässlich. Der Sprecher der SVP (Berger SVP/VD) bezeichnete die Koordination der Agrarproduktion in Nord und Süd als ein entscheidendes Element für ein weltweites Gleichgewicht.

Trotz angebrachter Selbstkritik sei die Entwicklungshilfe der letzten Jahre nicht nur negativ gewesen, sondern habe auch Entwicklungsfortschritte bewirkt. Selbstverständlich blieben grosse Probleme ungelöst und bedürften zusätzlicher Anstrengungen von beiden Seiten; Bundesrat Felber erwähnte in seinem Schlusswort beispielsweise die Stellung der Frau in der Dritten Welt.

\section{Aktivitäten}

Die politischen und kulturellen Aktivitäten konzentrierten sich auf die Monate März bis Juni 1988. Medien, Universitäten, Schulen, private Organisationen, die DEH, Radio und Fernsehen veranstalteten Debatten, Filme, Konzerte, Theateraufführungen und Ausstellungen. Dabei ging es um die Gesamtschau der Probleme, um den Zusammenhang von Handel, Verschuldung, Landwirtschaft, 
Beschäftigung, Ressourcen, Umwelt, Entwicklungshilfe und kulturelle Beziehungen. Es wurden Gewerkschafterinnen und Gewerkschafter, junge Menschen, Künstlerinnen und Künstler aus Entwicklungsländern in die Schweiz eingeladen.

Beispiele einiger Aktionen:

- Produkte-Informationsblätter erklärten die Herkunft von Rohstoffen und Konsumgütern im Rahmen der Wirtschaftsbeziehungen Schweiz-Dritte Welt.

- Ein Kulturmobil Nord/Süd animierte Projekte und Aktionen in den Dörfern und Quartieren.

- Ein Katalog des Forums "Schule für eine Welt» bot Lernziele, Leitideen und Richtlinien für die Erziehung zu einer globalen Weltsicht.

- Eine Medienbegegnung Nord/Süd mit einem Wettbewerb für TV-Sendungen zur Verbreitung und Förderung des Medienschaffens in der Dritten Welt fand in Genf statt.

- Medienschaffende aus Entwicklungsländern gestalteten Sendungen am Radio.

- "Negripub» - eine Ausstellung über das Bild der Schwarzen in der europäischen Produktewerbung - fand an der Universität Freiburg statt.

\section{Quellen}

Europarat, Erklärung von Madrid, Abschlussdokument der Konferenz von Madrid, 1.-3. Juni 1988

Europarat, Erklärung von Barcelona, Abschlussdokument der Konferenz von Barcelona, 30.-31. Mai 1988

Die Projekte der Kampagne Nord/Süd in der Schweiz, 3/88

Bbl 87.064 und 88.445

NZZ, 20.11.1987, 23.6.1988

Tages-Anzeiger, 4.6.1988 


\section{Fluchtkapital und illegale Finanzgeschäfte}

Philippinen: Die Guthaben des ehemaligen Präsidenten der Philippinen, Ferdinand Marcos, und seiner Anhänger, auf den Konten der Schweizer Banken sind bereits im März 1986 gesperrt worden. Im April 1986 wurde von der philippinischen Regierung bei den Schweizer Behörden ein Rechtshilfegesuch eingereicht. Am 1. Juli 1987 entschied das Bundesgericht, dass der Verdacht auf strafbare Handlungen des Ex-Präsidenten Marcos begründet war und dass die Blockierung seiner Guthaben zu Recht erfolgt sei. Das Gericht wies somit drei Beschwerden der Marcos-Familie ab, setzte aber gleichzeitig strenge Bedingungen für die Herausgabe von Unterlagen fest.

Im Januar 1988 richteten die Philippinen eine diplomatische Note an die betreffenden Kantone (Freiburg, Genf und Zürich). Die Note stellt fest, dass in den Philippinen ein Strafverfahren gegen Marcos eingeleitet wurde. Sie gibt ferner Auskunft über die Rekursrechte der Marcos-Anwälte. Eine Rechtshilfe kann in der Tat erst gewährt werden, wenn im Antragstellerland eine strafrechtliche Untersuchung eingeleitet wurde. Das Bundesgericht hatte ausserdem die Gewährleistung eines gerechten Urteils gefordert, insbesondere die Erlaubnis für den Ex-Präsidenten, für seine Verteidigung im Untersuchungsverfahren in sein Land zurückzukehren. In den Philippinen wird die Durchsetzung von Strafverfahren jedoch durch die politischen Probleme erschwert, die sich daraus ergeben würden, dass der Ex-Präsident in sein Land zurückkehrt, um sich den Richtern zu stellen. Eine solche Rückkehr scheint zur Zeit nicht erwünscht zu sein.

Die von Marcos bei acht Schweizer Banken in drei Kantonen hinterlegten Unterschlagungsgelder werden von der philippinischen Regierung auf 1,5 Milliarden Schweizer Franken geschätzt (1). Ferner sollen auch Vermögenswerte in Australien, Brasilien, in den Vereinigten Staaten, Liechtenstein, Panama und den Niederlanden deponiert worden sein. Die Gelder wurden häufig durch Mittelsmänner, unter dem Namen von Stiftungen oder anderen juristischen Personen, die ihr Domizil in der Schweiz oder in Liechtenstein hatten, verborgen. Dies erleichtert der Staatsanwaltschaft in den betroffenen Kantonen die Aufgabe keineswegs. In Zürich hat die Aufhebung des Bankgeheimnisses am meisten Zeit in Anspruch genommen. Diese Aufhebung soll es den Banken gemäss Urteil des Bundesgerichts erlauben, den Strafbehörden die nötigen Informationen zu liefern. Jedoch werden von der schweizerischen Gesetzgebung zahlreiche Rekursmöglichkeiten zum Schutz der Interessen der Konteninhaber vorgesehen. Diese Verfahren können eine aufschiebende Wirkung auf den Verlauf der Gerichtsverfahren haben. In Zürich wurden 32 Rekurse gegen die Weitergabe von Informationen über die angeklagten Konten an die kantonalen Justizbehörden eingereicht. In Genf und in Freiburg ist diese Phase rascher verlaufen. Die Justizbehörden des Kantons Freiburg konnten den philippinischen Gerichten bereits im Juni 1988 gewisse Bankunterlagen aushändigen. (Sie betreffen lediglich einen Betrag von 70 Millionen Franken.) 
In Zürich hatte die Schweizerische Kreditanstalt (SKA) bei der Staatsanwaltschaft gegen das Rechtshilfeverfahren Beschwerde eingelegt. Diese wies den Rekurs im Februar 1988 ab, indem sie sich auf das Urteil des Bundesgerichts stützte, bei dem die SKA in der Folge erneut Einspruch erhob. Nach Abweisung des Rekurses im Juni 1988 war die Schweizerische Kreditanstalt schliesslich gezwungen, den Zürcher Justizbehörden gewisse Bankunterlagen auszuhändigen. Dreissig weitere von Stiftungen und einem früheren Minister der Philippinen eingereichten Rekurse wurden von der Zürcher Staatsanwaltschaft im August 1988 abgewiesen.

Erst in einer späteren Phase entscheiden die Kantonsbehörden, dass den Justizbehörden der Philippinen bestimmte Dokumente ausgeliefert werden können (Kanton Freiburg), wobei beim Bundesgericht erneut Einspruch erhoben werden kann. Auch für die letzte Phase des Verfahrens, die Rückerstattung der Vermögenswerte an die philippinische Regierung, bestehen diese Einspruchsmöglichkeiten.

Es sei vermerkt, dass einige Hilfswerke, sowie linksgerichtete Kreise und Gruppen zur Unterstützung der Dritten Welt seit vielen Jahren eine Revision des Bankengesetzes verlangen, um jegliche Unterstützung, auch die passive Hilfe, bei der Kapitalflucht zu vermeiden und die Verpflichtungen der Banken zur Lieferung von Informationen an die Justizbehörden zu erweitern.

Anlässlich ihres Besuchs in der Schweiz, im Juni 1988, erklärte die philippinische Präsidentin Corazon Aquino, dass sie der Schweizer Regierung dankbar sei, die Marcos-Guthaben rasch gesperrt zu haben. Die Frage der Rückführung der Marcos-Gelder stand jedoch nicht offiziell auf der Tagesordnung der Gespräche mit den Bundesräten.

Im April 1988 haben die USA bei den Kantonen Genf und Zürich im Rahmen eines amerikanischen Strafverfahrens gegen Marcos ein Rechtshilfegesuch eingereicht. Dieses Verfahren steht im Zusammenhang mit 327 Millionen Dollar amerikanischer Wirtschaftshilfe, die zwischen 1978 und 1985 ausbezahlt wurden und von Marcos unterschlagen worden sein sollen. Der saudiarabische Waffenhändler Kashoggi soll bei Immobiliengeschäften und beim Kauf wertvoller Bilder als Strohmann gedient haben. Ferdinand Marcos und Adnan Kashoggi, sowie Imelda Marcos wurden am 21. Oktober 1988 in New York unter Anklage gestellt. Die USA streben an, in der Schweiz Informationen über die Veruntreuung dieser Entwicklungshilfegelder zu erhalten. Die Schweizer Bundesbehörden haben das Rechtshilfegesuch der Vereinigten Staaten im Oktober 1988 angenommen. Die Verteidigung kann ihrerseits wieder Einspruch einlegen und dabei bis zum Bundesgericht vorgehen.

Iran/Vereinigte Staaten: Die Finanzgeschäfte aus dem Waffenhandel zwischen den USA und dem Iran wurden über mehrere Bankkonten in der Schweiz (vor allem bei der Schweizerischen Kreditanstalt) durch Gesellschaften abgewickelt, von denen die meisten ihren Sitz in Gent hatten. Ein Teil der Erlöse aus diesem Waffengeschäft sollte an die Contra-Rebellen in Nicaragua überwiesen werden. 
Die amerikanische Regierung reichte in dieser Sache im Dezember 1986 ein Rechtshilfegesuch an die Schweiz ein. Daraufhin wurden die betroffenen Bankkonten gesperrt. Am 20. August 1987 hat das Bundesgericht die Blockierung der besagten Konten bestätigt. Die Genfer Anklagekammer hat die letzten Rekurse im September 1987 abgewiesen und dadurch die Herausgabe von Unterlagen an die amerikanischen Justizbehörden ermöglicht.

\section{Anmerkung}

1) Nach Ramon Diaz, Vorsitzender des Untersuchungsausschusses auf Regierungsebene in den Philippinen.

\section{Quellen}

Harrisson, Pierre. Etats-Unis contra Nicaragua. PubliCETIM No 16-17, 1988.

Journal de Genève, 20.1.1988, 31.8.1988.

Le Courrier, 19.8.1987 und 22./23.10.1988.

NZZ, 19.1., 23.2., 9.6., 13.6., 14.6., 16.6. und 16.8.1988.

Tages-Anzeiger, 23.2., 31.3., 21.5., 16.6., 11.7. und 22.10.1988. 